\title{
Trait Reappraisal Predicts Affective Reactivity to Daily Positive and Negative Events
}

\author{
Gul Gunaydin ${ }^{1 *}$,Emre Selcuk ${ }^{2}$ and Anthony D. Ong ${ }^{3}$ \\ ${ }^{1}$ Department of Psychology, Bilkent University, Ankara, Turkey, ${ }^{2}$ Department of Psychology, Middle East Technical University, \\ Ankara, Turkey, ${ }^{3}$ Department of Human Development, Cornell University, Ithaca, NY, USA
}

Past research on emotion regulation has provided evidence that cognitive reappraisal predicts reactivity to affective stimuli and challenge tests in laboratory settings. However, little is known about how trait reappraisal might contribute to affective reactivity to everyday positive and negative events. Using a large, life-span sample of adults $(N=1755)$, the present study addressed this important gap in the literature. Respondents completed a measure of trait reappraisal and reported on their daily experiences of positive and negative events and positive and negative affect for eight consecutive days. Results showed that trait reappraisal predicted lower increases in negative affect in response to daily negative events and lower increases in positive affect in response to daily positive events. These findings advance our understanding of the

OPEN ACCESS

Edited by:

John M. Zelenski,

Carleton University, Canada

Reviewed by:

Elizabeth K. Nisbet,

Trent University, Canada

Philippe Verduyn

Maastricht University, Netherlands

${ }^{*}$ Correspondence:

Gul Gunaydin

gulgunaydin@bilkent.edu.tr

Specialty section:

This article was submitted to Personality and Social Psychology,

a section of the journal

Frontiers in Psychology

Received: 01 April 2016

Accepted: 17 June 2016

Published: 28 June 2016

Citation:

Gunaydin G, Selcuk E and Ong AD (2016) Trait Reappraisal Predicts Affective Reactivity to Daily Positive and Negative Events.

Front. Psychol. 7:1000

doi: 10.3389/fpsyg.2016.01000 role of reappraisal in emotion regulation by showing how individual differences in the use of this strategy relate to emotional reactions to both positive and negative events outside the laboratory.

Keywords: reappraisal, affective reactivity, daily diary designs, emotion regulation, daily life, neuroticism

\section{INTRODUCTION}

Extant research on emotion regulation has demonstrated that cognitive reappraisal robustly predicts dampened reactivity to affective stimuli and stressful experiences in laboratory settings (Gross and John, 2003; John and Gross, 2004). Experimentally manipulating reappraisal by instructing participants "to adopt a detached and unemotional attitude" toward positive and negative photographs has been shown to be an effective regulation strategy for both positive and negative emotion (e.g., Gruber et al., 2014). Moreover, individual differences in reappraisal ability (i.e., trait reappraisal) are associated with less negative affect following stressful laboratory tasks such as anger provocation (e.g., Memedovic et al., 2010). To date, most research on reappraisal has been conducted in laboratory settings. Whether individual differences in reappraisal predicts affective reactivity to daily life events-especially pleasant experiences-has not been much studied. Although a large body of work has investigated how individuals appraise stressors (e.g., Lazarus and Folkman, 1987) and use various strategies to cope with them including mindfulness exercises (e.g., Grossman et al., 2004), expressive writing (e.g., Pennebaker, 1997), and reappraisal tactics (e.g., Ong et al., 2010), most of this work has not focused on daily affective reactivity. Establishing the link between trait reappraisal and daily affective reactivity is important because (a) the dynamics of affect in day-to-day life are different from those that arise in laboratory tasks and (b) recent prospective studies have shown that daily affective reactivity predicts important psychological and health outcomes-such as psychological well-being (Selcuk et al., 2016), sleep efficiency 
(Ong et al., 2013), inflammation (Sin et al., 2015), affective disorders (Peeters et al., 2003; Charles et al., 2013), and even mortality (Mroczek et al., 2015). Thus, the aim of the present study was to investigate affective reactivity more dynamically to complement single assessment surveys and laboratory research. To this end, we examined whether trait reappraisal predicts daily affective reactivity to both positive and negative events.

\section{Reappraisal and Daily Affective Reactivity}

Based on Gross's process model of emotion regulation (Gross, 2001), cognitive reappraisal is a strategy aimed at changing emotional reactions by re-interpreting emotional events or changing the way one thinks about events. Individuals might achieve this by re-evaluating emotional events in an objective, detached, and unemotional manner (e.g., Gross, 1998) or in a more positive light (e.g., Wrosch et al., 2000). Individual differences in reappraisal ability (i.e., trait reappraisal) were shown to modestly predict spontaneous use of reappraisal to cope with stressors. Specifically, individuals high in trait reappraisal also reported spontaneously using this strategy to a greater extent in stressful situations-such as giving a public speech or visiting the dentist (Egloff et al., 2006).

Given reappraisal involves changing the way one thinks about events, a natural implication of this strategy is that individuals might use reappraisal to up- or down-regulate both positive and negative affective reactions. Consistent with this idea, laboratory inductions of reappraisal have either instructed participants to up-regulate (Kim and Hamann, 2007; Ray et al., 2010) or downregulate (Winecoff et al., 2011; Gruber et al., 2014) affective reactions to positive or negative stimuli (e.g., film clips, pictures). This research demonstrates that participants are able to increase or decrease emotional responses to both positive and negative stimuli when instructed to do so. However, this work does not address the question of whether individuals high in trait reappraisal spontaneously tend to up-regulate or down-regulate emotional reactions to positive and negative experiences.

A few studies have examined the link between trait reappraisal and affective reactivity to laboratory stressors (Mauss et al., 2007; Memedovic et al., 2010; see also Ong et al., 2006). For example, in one study, individuals high (vs. low) in trait reappraisal reported experiencing less anger after receiving insulting feedback in the laboratory (Memedovic et al., 2010). Similarly, spontaneous use of a reappraisal strategy was also shown to be associated with reduced negative affect during a public speaking task (Egloff et al., 2006). Only a few recent studies have investigated the role of reappraisal in coping with day-to-day stressors. In an experience sampling study, Heiy and Cheavens (2014) asked individuals to report at random times throughout the day which strategies they used to regulate affective reactions and found that individuals who reported using reappraisal to regulate negative affect reported better current mood. However, this study focused on participants' introspections about emotion regulation strategies rather than the extent to which participants used reappraisal to regulate emotional reactions on a regular basis. Other research using similar methodology that controlled for the use of other emotion regulation strategies (e.g., reflection, distraction) failed to find a significant relationship between daily use of reappraisal and negative affect (Brans et al., 2013). However, this research focused on the predictive role of emotion regulation strategies in negative affect rather than in negative affective reactivity to stressors. Another study experimentally manipulated the daily use of reappraisal in response to the most negative event of the day over the course of a week (Ng and Diener, 2013). Results showed that participants who were instructed to reappraise the daily negative event reported lower negative affect about the event compared with participants who were instructed to focus on the event or those who did not try to regulate their emotions. However, this research focused on the instructed use of a reappraisal strategy rather than individual differences in reappraisal. Thus, although research in this area is growing, the paucity of current evidence necessitates continued investigations into the role of trait reappraisal in affective reactivity to daily negative events.

Research evidence linking trait reappraisal to reactions to positive events is even much more scant. Although one study found that individuals who reported using a reappraisal strategy to regulate positive affect did not experience significant changes in mood (Heiy and Cheavens, 2014), this study assessed spontaneous use of reappraisal rather than trait reappraisal. Moreover, unlike regulation of negative affect, it is unclear whether emotional reactions would be spontaneously up- or down-regulated in response to pleasant experiences. On the one hand, high reappraisers might re-interpret a daily positive event as even more pleasant, in which case they would experience greater positive affect (and less negative affect), which is considered an indicator of greater hedonic well-being (Lucas et al., 1996). On the other hand, there is evidence showing that greater positive affective reactivity (Peeters et al., 2003) and lower negative affective reactivity (Bylsma et al., 2011) to positive events are associated with greater depression. Moreover, recent research demonstrates that thinking about an emotional event from the perspective of an outside observer (vs. through one's own eyes), which is an emotion regulation strategy conducive to reappraisal, reduces the duration of affective experiences while reflecting on both negative and positive events (Verduyn et al., 2012). This work suggests that trait reappraisal might reduce reactivity not only to stressors but also to pleasant events. Therefore, further research is needed to understand how individuals high in trait reappraisal would respond to positive daily experiences.

\section{Present Research}

The aim of the present study was to address these important gaps in the literature by examining whether trait reappraisal predicts daily affective reactivity to both positive and negative events. Toward this aim, we used a large, life-span sample of adults $(N=1755)$ who completed a measure of trait reappraisal and reported on their daily experiences of positive and negative events as well as positive and negative affect for eight consecutive days. This allowed us to investigate whether trait reappraisal predicts changes in positive and negative affect from a day on which participants did not experience a negative (or a positive) event to a day on which they did experience such events (i.e., 
affective reactivity). We also aimed to investigate the unique role of trait reappraisal in affective reactivity by controlling for individual difference factors that prior work has shown to be linked with affective experience (e.g., Bolger and Zuckerman, 1995; Gunthert et al., 1999; Mroczek and Almeida, 2004; Leger et al., 2016). Toward this aim, we first tested in separate analyses whether age, gender, and neuroticism predicted daily positive and negative affective reactivity to positive and negative events. We then built two final analytical models, one for daily negative affect and one for daily positive affect. The final model for daily negative affect included all variables significantly associated with negative affective reactivity. Likewise, the final model for daily positive affect included all variables that were significantly associated with positive affective reactivity (see Leger et al., 2016 for a similar approach in modeling daily affective reactivity). This approach allowed us to control for potential confounds that may account for the associations between trait reappraisal and affective reactivity.

\section{MATERIALS AND METHODS}

\section{Sample and Procedures}

Data for the present study came from the second wave of the Midlife Development in the United States (MIDUS; Ryff et al., 2007) and the National Study of Daily Experiences (NSDE; Ryff and Almeida, 2010) projects. The MIDUS project was launched in $1994(N=7,108)$ to investigate age-related changes in physical and mental health of adults in the United States. MIDUS II ( $N=4,963$ ) was conducted in 2004-2006 as a 10-year followup on MIDUS I measures and included a phone interview followed by a self-administered questionnaire. Upon completion of MIDUS II, a subset of the MIDUS project participants were recruited to the NSDE II $(n=2,022)$ where they completed an 8-day diary study on common daily positive and negative events, and daily affect. A total of 1,772 adults completed both the MIDUS II and NSDE II studies. Of these, 17 (0.96\%) did not complete measures of reappraisal and/or neuroticism, leaving a final analytic sample of 1,755 adults (57\% females; mean age $=57$ years, range $=33-84$ years $)$. Mean time lag between the MIDUS II phone interview and the NSDE II was 21 months ( $S D=14$ months) in the current sample. The present sample was not significantly different than the remainder of the MIDUS II sample in terms of gender composition $\left(\chi^{2}=2.251, p=0.134\right)$ or mean age $(t=1.687, p=0.092)$. However, participants in the current sample scored slightly higher in reappraisal $(M=3.096$, $S E=0.014$ vs. $M=3.048, S E=0.013, t=2.464, p=0.014$, $d=0.079)$ and slightly lower in neuroticism $(M=2.057$, $S E=0.015$ vs. $M=2.122, S E=0.013, t=3.245, p=0.001$, $d=0.103)$.

Data collection was reviewed and approved by the Education and Social/Behavioral Sciences and the Health Sciences Institutional Review Boards (IRBs) at the University of Wisconsin-Madison. All participants provided verbal consent. The consent procedure assured participants that their participation was voluntary and that their data would be kept confidential. The IRBs approved the waiver of written consent.
Data and documentation for MIDUS II are publicly available at the Inter-university Consortium for Political and Social Research website $\left(\mathrm{ICPSR}^{1}\right)$.

\section{Measures}

\section{Trait Reappraisal}

Trait reappraisal was measured using the 4-item Positive Reappraisal Scale (Wrosch et al., 2000). Participants indicated the extent to which they used reappraisal strategies to cope with difficult situations (e.g., "When I am faced with a bad situation, it helps to find a different way of looking at things"). Participants responded to the items on a 4 -point Likert scale $(1=A$ lot to $4=$ Not at all). Items were reverse scored so that greater scores reflected higher trait reappraisal. A trait reappraisal score was calculated by averaging across the items $(M=3.096, S D=0.604$, $\alpha=0.78)$.

\section{Daily Positive and Negative Events}

On each of the 8 days during the NSDE II, participants completed a measure of common daily positive and negative events. Occurrence of daily negative events was measured using the Daily Inventory of Stressful Events (Almeida et al., 2002). This measure asks participants to indicate whether they had experienced any of the following common daily stressors: an interpersonal conflict, a situation that could end in an argument but they decided to avoid, a problem at work, a problem at home, something bad happening to a close other, perceived discrimination, and any other stressful experiences not covered by the previous categories. Participants also completed a measure of occurrence of daily positive events asking whether they had experienced any of the following events each day: a positive interaction with someone, a positive event at work, a positive event at home, something good happening to a close other, and any other pleasant events not covered by the previous categories. Mean number of stressors and positive events experienced per day was $0.53(S D=0.46)$ and $1.13(S D=0.67)$, respectively.

\section{Daily Affect}

Participants also indicated the frequency with which they had experienced several affective states each day $(0=$ None of the time to $4=$ All of the time). The items were adapted from the Non-Specific Psychological Distress and Positive Emotions Scale (Kessler et al., 2002). The items of the negative affect subscale included "restless or fidgety," "nervous," "worthless," "so sad nothing cheer you up," "everything was an effort," "hopeless," "lonely," “afraid," "jittery," "irritable," "ashamed," "upset," “angry," and "frustrated." (average $\alpha$ across days $=0.84$ ). The items of the positive affect subscale included "in good spirits," "cheerful," "extremely happy," "calm and peaceful," "satisfied," "full of life," "close to others," "like you belong," "enthusiastic," "attentive," "proud," "active," and "confident." (average $\alpha$ across days = 0.94). Because affect was measured at the day level, mean levels of positive and negative affect were estimated in a two-level null model. Mean daily negative affect was $0.191(S E=0.006)$ and mean daily positive affect was $2.727(S E=0.017)$.

\footnotetext{
${ }^{1}$ www.icpsr.umich.edu/icpsrweb
} 


\section{Neuroticism}

Neuroticism was measured using the Midlife Development Inventory Personality Scales (Lachman and Weaver, 1997), which were specifically developed for the MIDUS project. The scales were constructed using items from existing well-validated personality inventories, e.g., the Big Five Inventory (John, 1990). Participants were asked to indicate how much each item described them $(1=A$ lot to $4=$ Not at all $)$. The neuroticism subscale consisted of four items (moody, worrying, nervous, and calm). The item "nervous" overlaps with an item in the daily negative affect scale and the item "calm" overlaps with an item in the daily positive affect scale used in present research. To make sure that any relationship between neuroticism and daily affect was not due to item overlap, these two items were not used when calculating neuroticism scores (see Cacioppo et al., 2010 for a similar approach in estimating the association between loneliness and depression). Responses to the items "moody" and "worrying" were recoded and averaged so that higher scores reflected greater neuroticism $(M=2.086, S D=0.715, \alpha=0.61)$.

\section{Demographic Variables}

Participants reported their age and gender at the MIDUS II phone interview.

\section{Data Analytic Strategy}

Daily affective reactivity and its association with trait reappraisal was estimated using multilevel modeling (HLM v7 software). Following prior work (Mroczek et al., 2015; Selcuk et al., 2016), the following two-level model was used to estimate daily negative affect:

Level 1: Negative Affect $i j=\pi_{0 j}+\pi_{1 j}$ Negative Event $_{i j}+\pi_{2 j}$ Positive Event ${ }_{i j}+\mathrm{e}_{i j}$

Level 2: $\pi_{0 j}=\beta_{00}+\beta_{01}$ Person-Mean Negative Event ${ }_{j}+\beta_{01}$ Person-Mean Positive Event ${ }_{j}+\mathrm{r}_{0 j}$

$$
\begin{aligned}
& \pi_{1 j}=\beta_{10}+r_{1 j} \\
& \pi_{2 j}=\beta_{20}+r_{2 j}
\end{aligned}
$$

At Level $1, \pi_{0 j}$ is the intercept and represents negative affect experienced on a day when the participant did not experience a negative or a positive event. Negative Event was a dichotomous variable and was coded either as 0 (when no negative events were experienced) or as 1 (when at least one negative event was experienced). Hence, $\pi_{1 j}$ is the within-person affective reactivity slope corresponding to the difference in the participant's negative affect on days when at least one negative event was experienced compared to days when no negative events were experienced. In a similar vein, Positive Event was a dichotomous variable indicating whether the participant experienced any positive events that day and its coefficient, $\pi_{2 j}$, is the within-person affective reactivity slope corresponding to the difference in the participant's negative affect on days when at least one positive event was experienced compared to days when no positive events were experienced. The error term, $\mathrm{e}_{i j}$, represented the participant's deviation from his/her average negative affect. At Level 2, $\beta_{00}, \beta_{10}$, and $\beta_{20}$ represent the sample average of negative affect on no-negative event days, negative affective reactivity to stressors, and negative affective reactivity to positive events, respectively. Additionally, $\beta_{01}$ represents the association between person-mean frequency of negative event exposure and negative affect, and $\beta_{02}$ represents the association between person-mean frequency of positive events and negative affect. These terms were included in the model to control for between-person differences in negative and positive event exposure. Finally, the error terms, $\mathrm{r}_{0 j}, \mathrm{r}_{1 j}$, and $\mathrm{r}_{2 j}$ represented deviations from average negative affect, average negative affective reactivity to negative events, and average negative affectivity to positive events, respectively, in the entire sample. Daily positive affective reactivity was estimated in exactly the same way except that the outcome variable was positive affect.

Next, to examine whether reappraisal was associated with affective reactivity, we entered it into the model as a predictor of $\pi_{0 j}$ (estimating the main association of reappraisal with daily affect), $\pi_{1 j}$ (estimating the interaction of negative event exposure and trait reappraisal), and $\pi_{2 j}$ (estimating the interaction of positive event exposure and trait reappraisal). The Level 1 equation of the multilevel model was the same as above and the Level 2 equations were as follows:

$$
\begin{aligned}
\text { Level 2: } \pi_{0 j}= & \beta_{00}+\beta_{01} \text { Person-Mean Negative Event }{ }_{j}+ \\
& \beta_{02} \text { Person-Mean Positive Event }{ }_{j}+ \\
& \beta_{03} \text { Reappraisal }{ }_{j}+\mathrm{r}_{0 j} \\
& \pi_{1 j}=\beta_{10}+\beta_{11} \text { Reappraisal }+\mathrm{r}_{1 j} \\
& \pi_{2 j}=\beta_{20}+\beta_{21} \text { Reappraisal }+\mathrm{r}_{2 j}
\end{aligned}
$$

In the above model, $\beta_{11}$ and $\beta_{21}$ corresponded to change in affective reactivity to negative events and positive events, respectively, associated with one unit increase in trait reappraisal.

Finally, we examined whether the association between reappraisal and affective reactivity holds after controlling for other individual difference factors associated with daily affect. Following Leger et al. (2016), in separate analyses we first replaced reappraisal in the above Level 2 equations by each of the other individual difference factors (age, gender, and neuroticism) to test whether they predicted affective reactivity slopes. Then, we performed two final models, one for daily positive affect and one for daily negative affect. The final model for daily negative affect included all variables that were significantly associated with negative affective reactivity slopes. Similarly, the final model for daily positive affect included all variables that were significantly associated with positive affective reactivity slopes. In all of the multilevel models, continuous person-level (Level 2) variables were grand-mean centered and robust standard errors were used to estimate the confidence intervals for the model coefficients.

\section{RESULTS}

\section{Does Trait Reappraisal Predict Daily Negative Affective Reactivity?}

Multilevel modeling analyses revealed that experiencing a negative event was associated with an increase in negative affect $\left(\beta_{10}=0.165, p<0.001,95 \% \mathrm{CI}=[0.153,0.177]\right)$ whereas 
TABLE 1 | Multilevel models predicting daily negative affect.

\begin{tabular}{|c|c|c|c|c|c|c|}
\hline \multirow[b]{2}{*}{ Predictors } & \multicolumn{2}{|c|}{ Model 1} & \multicolumn{2}{|c|}{ Model 2} & \multicolumn{2}{|c|}{ Model 3} \\
\hline & Coefficient & $p$ & Coefficient & $p$ & Coefficient & $p$ \\
\hline \multicolumn{7}{|l|}{ Intercept, $\pi_{0}$} \\
\hline Intercept, $\beta_{00}$ & $0.123(0.006)$ & $<0.001$ & $0.123(0.006)$ & $<0.001$ & $0.121(0.008)$ & $<0.001$ \\
\hline Average negative events, $\beta_{01}$ & $0.158(0.016)$ & $<0.001$ & $0.155(0.016)$ & $<0.001$ & 0.139 (0.016) & $<0.001$ \\
\hline Average positive events, $\beta_{02}$ & $-0.041(0.008)$ & $<0.001$ & $-0.034(0.008)$ & $<0.001$ & $-0.031(0.008)$ & $<0.001$ \\
\hline Reappraisal, $\beta_{03}$ & - & - & $-0.026(0.010)$ & 0.010 & $-0.014(0.008)$ & 0.066 \\
\hline Age, $\beta_{04}$ & - & - & - & - & $0.001(0.0004)$ & 0.001 \\
\hline Gender, $\beta_{05}$ & - & - & - & - & $0.003(0.008)$ & 0.716 \\
\hline Neuroticism, $\beta_{06}$ & - & - & - & - & $0.054(0.008)$ & $<0.001$ \\
\hline \multicolumn{7}{|l|}{ Negative event slope, $\pi_{1}$} \\
\hline Intercept, $\beta_{10}$ & $0.165(0.006)$ & $<0.001$ & $0.165(0.006)$ & $<0.001$ & $0.149(0.007)$ & $<0.001$ \\
\hline Reappraisal, $\beta_{11}$ & - & - & $-0.033(0.010)$ & $<0.001$ & $-0.019(0.010)$ & 0.049 \\
\hline Age, $\beta_{12}$ & - & - & - & - & $-0.002(0.0005)$ & $<0.001$ \\
\hline Gender, $\beta_{13}$ & - & - & - & - & $0.024(0.011)$ & 0.022 \\
\hline Neuroticism, $\beta_{14}$ & - & - & - & - & $0.050(0.008)$ & $<0.001$ \\
\hline \multicolumn{7}{|l|}{ Positive event slope, $\pi_{2}$} \\
\hline Intercept, $\beta_{20}$ & $0.002(0.005)$ & 0.647 & 0.002 (0.005) & 0.686 & $0.002(0.005)$ & 0.732 \\
\hline Reappraisal, $\beta_{21}$ & - & - & $-0.005(0.008)$ & 0.550 & - & - \\
\hline Age, $\beta_{22}$ & - & - & - & - & $-0.001(0.0004)$ & 0.030 \\
\hline
\end{tabular}

Standard errors are provided in parantheses. All continuous variables were grand-mean centered. For gender, male was coded as 0 and female was coded as 1.

experiencing a positive event was not significantly associated with negative affect $\left(\beta_{20}=0.002, p=0.647,95 \% \mathrm{CI}=[-0.008,0.012]\right.$; see Model 1 of Table 1). At Level 2, person-mean negative event exposure was positively $\left(\beta_{01}=0.158, p<0.001,95 \% \mathrm{CI}=[0.127\right.$, $0.189]$ ) and person-mean positive event exposure was negatively associated with daily negative affect $\left(\beta_{02}=-0.041, p<0.001\right.$, $95 \% \mathrm{CI}=[-0.057,-0.025])$, indicating that individuals who on average experience a greater number of stressors and fewer number of pleasant events experience higher daily negative affect. Trait reappraisal was also associated with lower negative affect $\left(\beta_{02}=-0.026, p=0.010,95 \% \mathrm{CI}=[-0.046,-0.06]\right.$; Model 2 of Table 1). It also predicted lower negative affective reactivity to negative event exposure $\left(\beta_{11}=-0.033, p<0.001\right.$, $95 \%$ CI $=[-0.051,-0.015])$. That is, individuals high in reappraisal experienced lower increases in negative affect from a no-stressor day to a day on which they experienced at least one stressful event (Figure 1). However, trait reappraisal did not significantly predict negative affective reactivity to positive events $\left(\beta_{21}=-0.005, p=0.550,95 \% \mathrm{CI}=[-0.021,0.011]\right)$. Age, gender, and neuroticism were significantly associated with negative affective reactivity to stressful events (all $p s<0.005$ ), and age was significantly associated with negative affective reactivity to positive events $(p=0.037)$. Thus, we performed a final model including person-mean negative events, personmean positive events, reappraisal, neuroticism, age, and gender as predictors of average negative affect (i.e., intercept); trait reappraisal, age, gender, and neuroticism as predictors of negative affective reactivity to stressors; and age as the predictor of negative affective reactivity to positive events. In this final model being female, younger, and higher on neuroticism significantly predicted greater negative affective reactivity to negative events. Trait reappraisal was still significantly associated with negative

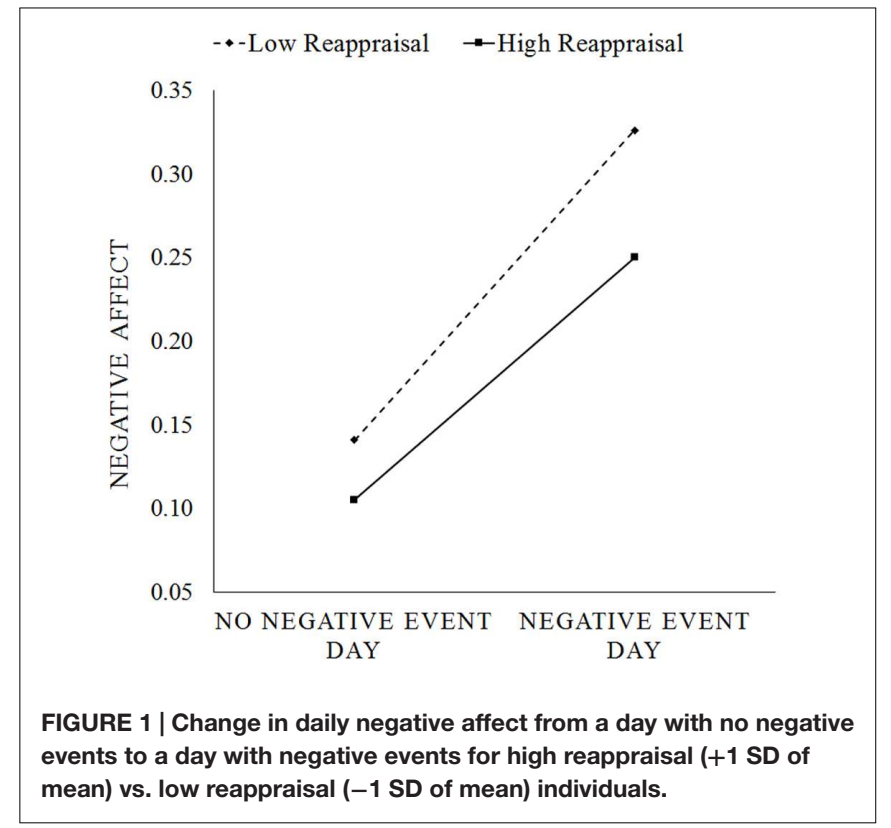

affective reactivity to negative events in the final model $\left(\beta_{11}=-0.019, p=0.049,95 \% \mathrm{CI}=[-0.038,-0.0001]\right)$. Age was also negatively associated with negative affective reactivity to positive events (see Model 3 of Table 1 for all coefficients).

\section{Does Trait Reappraisal Predict Daily Positive Affective Reactivity?}

At the within-person level, exposure to a positive event was associated with increases $\left(\beta_{20}=0.081, p<0.001,95 \%\right.$ 
TABLE 2 | Multilevel models predicting daily positive affect.

\begin{tabular}{|c|c|c|c|c|c|c|}
\hline \multirow[b]{2}{*}{ Predictors } & \multicolumn{2}{|c|}{ Model 1} & \multicolumn{2}{|c|}{ Model 2} & \multicolumn{2}{|c|}{ Model 3} \\
\hline & Coefficient & $p$ & Coefficient & $p$ & Coefficient & $p$ \\
\hline \multicolumn{7}{|l|}{ Intercept, $\pi_{0}$} \\
\hline Intercept, $\beta_{00}$ & $2.723(0.019)$ & $<0.001$ & $2.726(0.018)$ & $<0.001$ & $2.714(0.026)$ & $<0.001$ \\
\hline Average negative events, $\beta_{01}$ & $-0.521(0.041)$ & $<0.001$ & $-0.492(0.040)$ & $<0.001$ & $-0.409(0.040)$ & $<0.001$ \\
\hline Average positive events, $\beta_{02}$ & $0.240(0.026)$ & $<0.001$ & $0.169(0.026)$ & $<0.001$ & $0.142(0.025)$ & $<0.001$ \\
\hline Reappraisal, $\beta_{03}$ & - & - & $0.339(0.031)$ & $<0.001$ & $0.291(0.030)$ & $<0.001$ \\
\hline Age, $\beta_{04}$ & - & - & - & - & $0.003(0.001)$ & 0.006 \\
\hline Gender, $\beta_{05}$ & - & - & - & - & $0.021(0.036)$ & 0.549 \\
\hline Neuroticism, $\beta_{06}$ & - & - & - & - & $-0.183(0.023)$ & $<0.001$ \\
\hline \multicolumn{7}{|l|}{ Negative event slope, $\pi_{1}$} \\
\hline Intercept, $\beta_{10}$ & $-0.143(0.009)$ & $<0.001$ & $-0.143(0.009)$ & $<0.001$ & $-0.111(0.012)$ & $<0.001$ \\
\hline Reappraisal, $\beta_{11}$ & - & - & $0.008(0.014)$ & 0.576 & - & - \\
\hline Gender, $\beta_{12}$ & - & - & - & - & $-0.056(0.017)$ & 0.001 \\
\hline \multicolumn{7}{|l|}{ Positive event slope, $\pi_{2}$} \\
\hline Intercept, $\beta_{20}$ & $0.081(0.010)$ & $<0.001$ & $0.079(0.010)$ & $<0.001$ & $0.055(0.013)$ & $<0.001$ \\
\hline Reappraisal, $\beta_{21}$ & - & - & $-0.036(0.017)$ & 0.037 & $-0.037(0.017)$ & 0.031 \\
\hline Gender, $\beta_{22}$ & & & - & - & $0.041(0.020)$ & 0.035 \\
\hline
\end{tabular}

Standard errors are provided in parantheses. All continuous variables were grand-mean centered. For gender, male was coded as 0 and female was coded as 1.

$\mathrm{CI}=[0.061,0.101])$ and exposure to a negative event was associated with decreases in daily positive affect $\left(\beta_{10}=-0.143\right.$, $p<0.001,95 \%$ CI $=[-0.161,-0.125]$; Model 1 of Table 2). There was also between-person differences in the links between daily events and positive affect. Individuals who experienced on average greater number of positive events and fewer number of negative events experienced higher daily positive affect $\left(\beta_{02}=0.240, p<0.001,95 \%\right.$ $\mathrm{CI}=[0.189,0.291] ; \quad \beta_{01}=-0.521, p<0.001,95 \%$ $\mathrm{CI}=[-0.601,-0.441$, respectively $]$. Trait reappraisal also positively predicted daily positive affect $\left(\beta_{03}=0.339, p<0.001\right.$, 95\% CI $=[0.278,0.400]$; Model 2 of Table 2). Importantly, reappraisal negatively predicted positive affective reactivity to positive events $\left(\beta_{21}=-0.036, p=0.037,95 \% \mathrm{CI}=[-0.069\right.$, $-0.003])$. That is, high reappraisal individuals experienced lower increases in positive affect from a no-positive event day to a day in which they experienced at least one positive event (Figure 2). However, trait reappraisal did not predict positive affective reactivity to negative events $\left(\beta_{11}=0.008\right.$, $95 \% \mathrm{CI}=[-0.019,0.035]$. Although gender was significantly associated with positive affective reactivity to both positive events $(p=0.050)$ and negative events $(p<0.001)$, neither age nor neuroticism was significantly associated with positive affective reactivity to positive or negative events (all $p s>0.064$ ). Thus, we performed a final model including person-mean negative events, person-mean positive events, reappraisal, neuroticism, age, and gender as predictors of average positive affect (i.e., intercept), gender as the predictor of positive affective reactivity to negative events, and reappraisal and gender as predictors of positive affective reactivity to positive events. In the final model, reappraisal still significantly predicted positive affective reactivity to positive events $\left(\beta_{21}=-0.037, p=0.031,95 \% \mathrm{CI}=[-0.070\right.$, $-0.004]$ ). Gender also significantly predicted positive affective reactivity, with female (vs. male) participants' positive affective

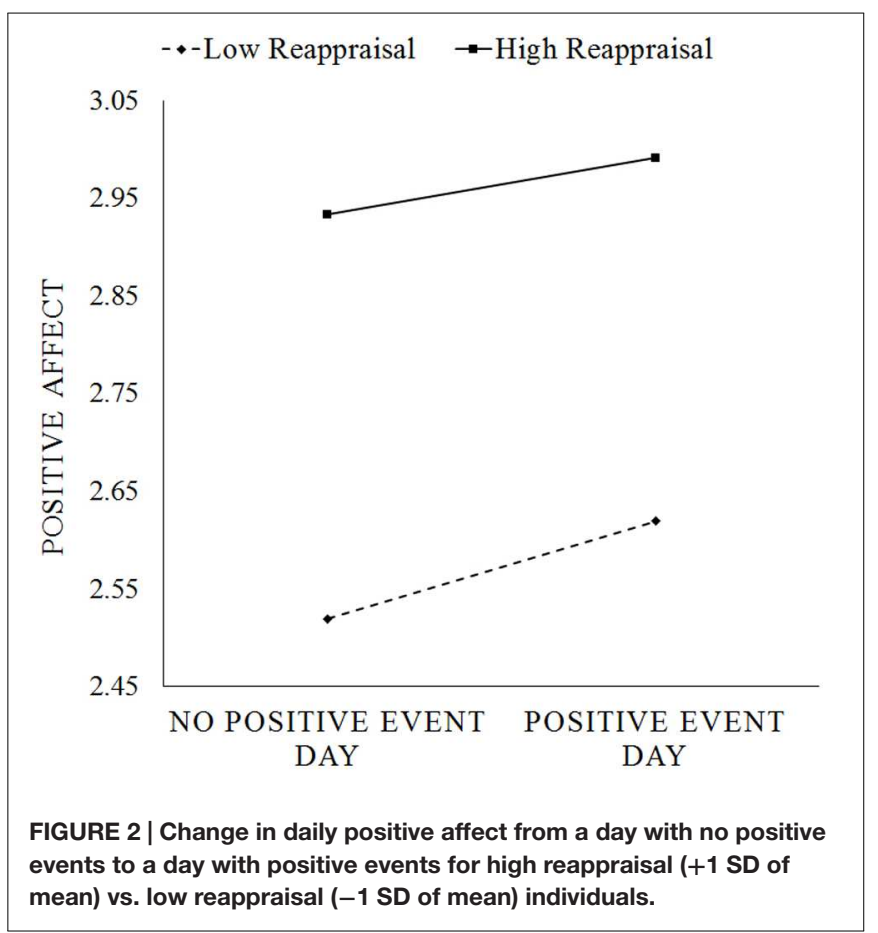

reactivity being higher in response to positive events and lower in response to negative events (see Model 3 of Table 2 for all coefficients).

\section{DISCUSSION}

Using multilevel analyses of daily experience data from a life-span sample of adults, the present research showed that 
trait reappraisal predicted lower increases in negative affect in response to daily negative events. However, trait reappraisal did not significantly predict change in negative affect in response to positive events. The association between trait reappraisal and negative affective reactivity to stressors held controlling for other significant predictors of negative affective reactivity (i.e., age, gender, and neuroticism). These findings contribute to the literature in affect regulation by showing the link between trait reappraisal and negative affective reactivity to stressors in daily life. This is a small but practically important effect given that daily affective reactivity is known to play a central role in psychological and physical well-being-from how fulfilled one's life is (Selcuk et al., 2016), to how well one sleeps (Ong et al., 2013), and ultimately to how long one lives (Mroczek et al., 2015).

The present research also showed that trait reappraisal predicted lower increases in positive affect in response to daily positive (but not negative) events. Although this is a small association, it significantly contributes to theorizing on reappraisal by clarifying whether individual differences in trait reappraisal would be associated with up- or downregulation of positive affect in response to pleasant events. Given previous studies investigating affective reactivity to positive stimuli almost exclusively focused on the instructed use of the reappraisal strategy (e.g., Gruber et al., 2014), little is known about how individual differences in the use of this strategy relate to affective reactivity to positive stimuli or pleasant daily experiences. Our findings demonstrate that individual differences in reappraisal predict down-regulation of positive affect in response to daily positive events, consistent with recent evidence showing that reappraising a positive affect-eliciting event by adopting an observer's perspective is associated with shorter durations of positive affect (Verduyn et al., 2012). These findings have important clinical implications given that past research has demonstrated that downregulation of positive affect in response to daily positive events is associated with better mental health (Peeters et al., 2003).

Another important implication of the present research is for understanding potential mechanisms explaining the wellestablished link between trait reappraisal and psychological adjustment. Specifically, trait reappraisal has been shown to predict lower depression (Garnefski and Kraaij, 2006; Aldao et al., 2010), higher self-esteem (Nezlek and Kuppens, 2008), greater life satisfaction, and better interpersonal relationships (Gross and John, 2003). However, mechanisms explaining these associations have not received much research attention. Our findings suggest that daily affective reactivity might be one of the potential mechanisms explaining the link between higher reappraisal ability and psychological adjustment.

To understand whether the use of affect regulation strategiesincluding reappraisal-might alter individual differences in daily affective reactivity one important question is: how stable are these individual differences? On the one hand, there is reason to expect stability. Research showed that affective reactivity is linked with relatively stable characteristics-such as activation in frontal regions of the brain (Davidson, 1992) and recollections of childhood experiences (Mallers et al., 2010). On the other hand, there is evidence that contextual factorssuch as how overwhelming and uncontrollable individuals view current life demands (Sliwinski et al., 2009) or how responsive they see close relationship partners (Selcuk et al., 2016)-predict affective reactivity. This work suggests that individuals' affective reactivity might change if their personal circumstances change. Indeed, research using the MIDUS sample showed modest stability $(r=0.37)$ in negative affective reactivity over 10 years (Sliwinski et al., 2009). In other words, although affective reactivity demonstrates some stability over time, there is also room for change. Therefore, it might be possible to alter one's affective reactivity by repeatedly using adaptive emotion regulation strategies such as reappraising or re-interpreting events (e.g., Gross, 2001), viewing them from a distanced perspective (e.g., Ayduk and Kross, 2010), activating mental representations of significant others (e.g., Selcuk et al., 2012), exercising mindfulness (e.g., Grossman et al., 2004), or writing about one's deepest thoughts and feelings about emotional events (e.g., Pennebaker, 1997). These are undoubtedly important questions for future research.

In sum, the present research is the first to document the link between trait reappraisal and affective reactivity to both positive and negative experiences in everyday life. Notably, the large sample spanning from middle to late adulthood increases the confidence in the generalizability of the findings. This work advances our understanding of the role of reappraisal in emotion regulation by showing how individual differences in the use of this strategy relate to emotional reactions to both stressors and pleasant events outside the laboratory and by offering a potential mechanism that may explain why trait reappraisal is associated with better psychological adjustment.

\section{AUTHOR CONTRIBUTIONS}

GG, ES, and AO developed the study idea. ES and GG conceived the data analytic strategy. ES conducted the data analysis. GG drafted the manuscript, and ES and AO provided critical revisions. All authors approved the final version of the manuscript for submission.

\section{FUNDING}

The MIDUS II research was supported by a grant from the National Institute on Aging (P01-AG020166) to conduct a longitudinal follow-up of the MIDUS I investigation. 


\section{REFERENCES}

Aldao, A., Nolen-Hoeksema, S., and Schweizer, S. (2010). Emotion-regulation strategies across psychopathology: a meta-analytic review. Clin. Psychol. Rev. 30, 217-237. doi: 10.1016/j.cpr.2009.11.004

Almeida, D. M., Wethington, E., and Kessler, R. C. (2002). The daily inventory of stressful events: an interview-based approach for measuring daily stressors. Assessment 9, 41-55. doi: 10.1177/1073191102091006

Ayduk, Ö., and Kross, E. (2010). Analyzing negative experiences without ruminating: the role of self-distancing in enabling adaptive self-reflection. Soc. Pers. Psychol. Compass 4, 841-854. doi: 10.1111/j.1751-9004.2010. 00301.x

Bolger, N., and Zuckerman, A. (1995). A framework for studying personality in the stress process. J. Pers. Soc. Psychol. 69, 890-902. doi: 10.1037/00223514.69.5.890

Brans, K., Koval, P., Verduyn, P., Lim, Y. L., and Kuppens, P. (2013). The regulation of negative and positive affect in daily life. Emotion 13, 926-939. doi: $10.1037 / \mathrm{a} 0032400$

Bylsma, L. M., Taylor-Clift, A., and Rottenberg, J. (2011). Emotional reactivity to daily events in major and minor depression. J. Abnorm. Psychol. 120, 155-167. doi: $10.1037 / \mathrm{a} 0021662$

Cacioppo, J. T., Hawkley, L. C., and Thisted, R. A. (2010). Perceived social isolation makes me sad: 5-year cross-lagged analyses of loneliness and depressive symptomatology in the Chicago Health, Aging, and Social Relations Study. Psychol. Aging 25, 453-463. doi: 10.1037/a0017216

Charles, S. T., Piazza, J. R., Mogle, J., Sliwinski, M. J., and Almeida, D. M. (2013). The wear and tear of daily stressors on mental health. Psychol. Sci. 24, 733-741. doi: $10.1177 / 0956797612462222$

Davidson, R. J. (1992). Emotion and affective style: hemispheric substrates. Psychol. Sci. 3, 39-43. doi: 10.1111/j.1467-9280.1992.tb00254.x

Egloff, B., Schmukle, S. C., Burns, L. R., and Schwerdtfeger, A. (2006). Spontaneous emotion regulation during evaluated speaking tasks: associations with negative affect, anxiety expression, memory, and physiological responding. Emotion 6, 356-366. doi: 10.1037/1528-3542.6.3.356

Garnefski, N., and Kraaij, V. (2006). Relationships between cognitive emotion regulation strategies and depressive symptoms: a comparative study of five specific samples. Pers. Individ. Dif. 40, 1659-1669. doi: 10.1016/j.paid.2005.12.009

Gross, J. J. (1998). Antecedent- and response-focused emotion regulation: divergent consequences for experience, expression, and physiology. J. Pers. Soc. Psychol. 74, 224-237. doi: 10.1037/0022-3514.74.1.224

Gross, J. J. (2001). Emotion regulation in adulthood: timing is everything. Curr. Dir. Psychol. Sci. 10, 214-219. doi: 10.1111/1467-8721.00152

Gross, J. J., and John, O. P. (2003). Individual differences in two emotion regulation processes: implications for affect, relationships, and well-being. J. Pers. Soc. Psychol. 85, 348-362. doi: 10.1037/0022-3514.85.2.348

Grossman, P., Niemann, L., Schmidt, S., and Walach, H. (2004). Mindfulnessbased stress reduction and health benefits: a meta-analysis. J. Psychosom. Res. 57, 35-43. doi: 10.1016/S0022-3999(03)00573-7

Gruber, J., Hay, A. C., and Gross, J. J. (2014). Rethinking emotion: cognitive reappraisal is an effective positive and negative emotion regulation strategy in bipolar disorder. Emotion 14, 388-396. doi: 10.1037/a0035249

Gunthert, K. C., Cohen, L. H., and Armeli, S. (1999). The role of neuroticism in daily stress and coping. J. Pers. Soc. Psychol. 77, 1087-1100. doi: 10.1037/00223514.77.5.1087

Heiy, J. E., and Cheavens, J. S. (2014). Back to basics: a naturalistic assessment of the experience and regulation of emotion. Emotion 14, 878-891. doi: $10.1037 / \mathrm{a} 0037231$

John, O. P. (1990). "The "Big Five" factor taxonomy: dimensions of personality in the natural language and in questionnaires," in Handbook of Personality: Theory and Research, ed. L. A. Pervin (New York, NY: Guilford Press), 66-100.

John, O. P., and Gross, J. J. (2004). Healthy and unhealthy emotion regulation: personality processes, individual differences, and life span development. J. Pers. 72, 1301-1333. doi: 10.1111/j.1467-6494.2004.00298.x

Kessler, R. C., Andrews, G., Colpe, L. J., Hiripi, E., Mroczek, D. K., Normand, S. L. T., et al. (2002). Short screening scales to monitor population prevalences and trends in non-specific psychological distress. Psychol. Med. 32, 959-976. doi: $10.1017 /$ S0033291702006074
Kim, S., and Hamann, S. (2007). Neural correlates of positive and negative emotion regulation. J. Cogn. Neurosci. 19, 776-798. doi: 10.1162/jocn.2007.19.5.776

Lachman, M. E., and Weaver, S. L. (1997). The Midlife Development Inventory (MIDI) Personality Scales: Scale Construction and Scoring. Waltham, MA: Brandeis University.

Lazarus, R. S., and Folkman, S. (1987). Transactional theory and research on emotions and coping. Eur. J. Pers. 1, 141-169. doi: 10.1002/per.2410010304

Leger, K. A., Charles, S. T., Turiano, N. A., and Almeida, D. M. (2016). Personality and stressor related affect. J. Pers. Soc. Psychol. doi: 10.1037/pspp0000083 [Epub ahead of print].

Lucas, R. E., Diener, E., and Suh, E. (1996). Discriminant validity of well-being measures. J. Pers. Soc. Psychol. 71, 616-628. doi: 10.1037/0022-3514.71.3.616

Mallers, M. H., Charles, S. T., Neupert, S. D., and Almeida, D. M. (2010). Perceptions of childhood relationships with mother and father: daily emotional and stressor experiences in adulthood. Dev. Psychol. 46, 1651-1661. doi: $10.1037 / \mathrm{a} 0021020$

Mauss, I. B., Cook, C. L., Cheng, J. Y. J., and Gross, J. J. (2007). Individual differences in cognitive reappraisal: experiential and physiological responses to an anger provocation. Int. J. Psychophysiol. 66, 116-124. doi: 10.1016/j.ijpsycho.2007.03.017

Memedovic, S., Grisham, J. R., Denson, T. F., and Moulds, M. L. (2010). The effects of trait reappraisal and suppression on anger and blood pressure in response to provocation. J. Res. Pers. 44, 540-543. doi: 10.1016/j.jrp.2010.05.002

Mroczek, D. K., and Almeida, D. M. (2004). The effect of daily stress, personality, and age on daily negative affect. J. Pers. 72, 355-378. doi: 10.1111/j.00223506.2004.00265.x

Mroczek, D. K., Stawski, R. S., Turiano, N. A., Chan, W., Almeida, D. M., Neupert, S. D., et al. (2015). Emotional reactivity and mortality: longitudinal findings from the VA normative aging study. J. Gerontol. B Psychol. Sci. Soc. Sci. 70, 398-406. doi: 10.1093/geronb/gbt107

Nezlek, J. B., and Kuppens, P. (2008). Regulating positive and negative emotions in daily life. J. Pers. 76, 561-580. doi: 10.1111/j.1467-6494.2008.00496.x

Ng, W., and Diener, E. (2013). Daily use of reappraisal decreases negative emotions toward daily unpleasant events. J. Soc. Clin. Psychol. 32, 530-545. doi: 10.1521/jscp.2013.32.5.530

Ong, A., Exner-Cortens, D., Riffin, C., Steptoe, A., Zautra, A., and Almeida, D. (2013). Linking stable and dynamic features of positive affect to sleep. Ann. Behav. Med. 46, 52-61. doi: 10.1007/s12160-013-9484-8

Ong, A. D., Bergeman, C. S., Bisconti, T. L., and Wallace, K. A. (2006). Psychological resilience, positive emotions, and successful adaptation to stress in later life. J. Pers. Soc. Psychol. 91, 730-749. doi: 10.1037/0022-3514.91. 4.730

Ong, A. D., Fuller-Rowell, T. E., and Bonanno, G. A. (2010). Prospective predictors of positive emotions following spousal loss. Psychol. Aging 25, 653-660. doi: $10.1037 / \mathrm{a} 0018870$

Peeters, F., Nicolson, N. A., Berkhof, J., Delespaul, P., and deVries, M. (2003). Effects of daily events on mood states in major depressive disorder. J. Abnorm. Psychol. 112, 203-211. doi: 10.1037/0021-843X.112.2.203

Pennebaker, J. W. (1997). Writing about emotional experiences as a therapeutic process. Psychol. Sci. 8, 162-166. doi: 10.1111/j.1467-9280.1997.tb00403.x

Ray, R. D., McRae, K., Ochsner, K. N., and Gross, J. J. (2010). Cognitive reappraisal of negative affect: converging evidence from EMG and self-report. Emotion 10, 587-592. doi: 10.1037/a0019015

Ryff, C. D., and Almeida, D. M. (2010). National Survey of Midlife in the United States (MIDUS II): Daily Stress Project, 2004-2009. ICPSR26841-v1. Ann Arbor, MI: Inter-university Consortium for Political and Social Research, $02-26$.

Ryff, C. D., Almeida, D. M., Ayanian, J. S., Carr, D. S., Cleary, P. D., Coe, C., et al. (2007). Midlife Development in the United States (MIDUS II), 2004-2006. Ann Arbor, MI: Inter-University Consortium for Political and Social Research.

Selcuk, E., Gunaydin, G., Ong, A. D., and Almeida, D. M. (2016). Does partner responsiveness predict hedonic and eudaimonic well-being? A 10year longitudinal study. J. Marriage Fam. 78, 311-325. doi: 10.1111/jomf. 12272

Selcuk, E., Zayas, V., Gunaydin, G., Hazan, C., and Kross, E. (2012). Mental representations of attachment figures facilitate recovery following upsetting autobiographical memory recall. J. Pers. Soc. Psychol. 103, 362-378. doi: $10.1037 / \mathrm{a} 0028125$ 
Sin, N. L., Graham-Engeland, J. E., Ong, A. D., and Almeida, D. M. (2015). Affective reactivity to daily stressors is associated with elevated inflammation. Health Psychol. 34, 1154-1165. doi: 10.1037/hea0000240

Sliwinski, M. J., Almeida, D. M., Smyth, J., and Stawski, R. S. (2009). Intraindividual change and variability in daily stress processes: findings from two measurement-burst diary studies. Psychol. Aging 24, 828-840. doi: $10.1037 / \mathrm{a} 0017925$

Verduyn, P., Van Mechelen, I., Kross, E., Chezzi, C., and Van Bever, F. (2012). The relationship between self-distancing and the duration of negative and positive emotional experiences in daily life. Emotion 12, 1248-1263. doi: $10.1037 / \mathrm{a} 0028289$

Winecoff, A., LaBar, K. S., Madden, D. J., Cabeza, R., and Huettel, S. A. (2011). Cognitive and neural contributors to emotion regulation in aging. Soc. Cogn. Affect. Neurosci. 6, 165-176. doi: 10.1093/scan/nsq030
Wrosch, C., Heckhausen, J., and Lachman, M. E. (2000). Primary and secondary control strategies for managing health and financial stress across adulthood. Psychol. Aging 15, 387-399. doi: 10.1037/0882-7974.15.3.387

Conflict of Interest Statement: The authors declare that the research was conducted in the absence of any commercial or financial relationships that could be construed as a potential conflict of interest.

Copyright (c) 2016 Gunaydin, Selcuk and Ong. This is an open-access article distributed under the terms of the Creative Commons Attribution License (CC BY). The use, distribution or reproduction in other forums is permitted, provided the original author(s) or licensor are credited and that the original publication in this journal is cited, in accordance with accepted academic practice. No use, distribution or reproduction is permitted which does not comply with these terms. 